Editorial

\title{
Comment on "Systematic Review and Meta-analysis of Efficacy and Safety of Melatonin and Triclofos for Inducing Adequate Sedation for sleep EEG in Children"
}

\author{
Amit Agrawal ${ }^{1}$ \\ 1 Department of Pediatrics, Gandhi Medical College \& Kamla Nehru \\ Hospital, Bhopal, Madhya Pradesh, India
}

J Neurosci Rural Pract 2022;13:1-2.

Sleep state is vital for recording electroencephalogram (EEG), as it decreases the artifacts and can unwind certain epileptiform discharges (EDs). However, attaining the sleep state in children, especially in children with developmental challenges, is often tricky. To achieve this sleep state in uncooperative patients, both children and adults, oral sedatives are used for prompting sleep, in order to assist in performing EEG. Sedation can be defined as a state of drowsiness or sleep from which a patient can be roused. ${ }^{1}$ In a sedated state, patients' vital respiratory reflexes remain intact, and they can respond to verbal commands. Scottish Intercollegiate Guidelines Network guideline is a national clinical guideline developed to highlight the special considerations for sedation in children. ${ }^{2}$

Various medications such as benzodiazepines, barbiturates, etc., have been tried in the past to achieve sedation. However, these drugs were noted to affect EEG recordings by either warping the background readings or stifling the EDs. Therefore, natural sleep was preferred for performing EEGs. To achieve this natural sleep state in uncooperative children, drugs such as chloral hydrate, chlorpromazine, and promethazine were previously favored. However, these drugs also lost their popularity over time due to their unacceptable adverse drug effects (ADE) profiles like prolonged sedation, respiratory depression, hypotension, and desaturation in certain cases.

This lead to the advent of drugs with better safety profiles such as melatonin, chloral hydrate, and triclofos. Melatonin is a hormone secreted by the pineal gland that acts primarily to regulate the circadian rhythm in humans. It is secreted at a serum concentration, varying extensively between 80 to 120 $\mathrm{pg} / \mathrm{mL}$ during the night and 10 to $20 \mathrm{pg} / \mathrm{mL}$ during the day. ${ }^{3}$ Therefore, it can be considered as one of the best biomarkers of human biorhythms. ${ }^{4}$ Children with neurodevelopmental disorders present with sleep problems that are mostly related to behavioral factors and altered circadian rhythm due to abnormally low levels of melatonin. ${ }^{5}$ Over the years, various researches have given abundant evidence that exogenous melatonin administration is effective against sleep problems. ${ }^{5,6}$ Moreover, being an indigenous hormone, melatonin causes a minimal adverse reaction in humans when compared with benzodiazepines, barbiturates, and propofol. Wassmer et al conducted a study to evaluate the efficacy and safety of melatonin for inducing sleep and suggested that melatonin is an efficacious drug to induce sedation for various noninvasive procedures in adults, children, and even in those with developmental or behavioral challenges. ${ }^{7}$ Although the adverse effects of melatonin are minor, it causes fatigue, mood swings, and tainted psychomotor performances to a certain extent.

Similarly, chloral hydrate derivatives are another group of drugs that are commonly used as oral sedatives. These drugs get metabolized to trichloroethanol. Since chloral hydrate has an unpleasant taste and causes gastric irritation, triclofos, a chloral hydrate derivative, is preferred as it is more palatable. The oral solution is well-absorbed, with the onset of action in 30 to 40 minutes, and produces hypnosis for 6 to 8 hours in doses of 25 to $75 \mathrm{mg} / \mathrm{kg}^{8}{ }^{8}$

In this issue of the Journal of Neurosciences in Rural Practice, Panda et al have published a systematic review and meta-analysis to assess the efficacy and safety of melatonin and triclofos for inducing adequate sedation for sleep EEG in children. ${ }^{9}$ Their initial searches led to the retrieval of 203 articles that highlighted the use of either melatonin or triclofos for sedation of children to perform EEGs. However, after a thorough evaluation of the articles by the reviewers, only 16 articles met the inclusion criteria (11 studies describing the efficacy of melatonin and 5 studies describing
Address for correspondence Amit Agrawal, MD, 28, Ravidas Nagar, Near Nizamuddin Colony, Indrapuri, Bhopal, Madhya Pradesh 462023, India (e-mail: agrawaldramit@yahoo. co.in).
DOI https://doi.org/ 10.1055/s-0041-1740579. ISSN 0976-3147.

(c) 2022. Association for Helping Neurosurgical Sick People. All rights reserved.

This is an open access article published by Thieme under the terms of the Creative Commons Attribution-NonDerivative-NonCommercial-License, permitting copying and reproduction so long as the original work is given appropriate credit. Contents may not be used for commercial purposes, or adapted, remixed, transformed or built upon. (https://creativecommons.org/ licenses/by-nc-nd/4.0/)

Thieme Medical and Scientific Publishers Pvt. Ltd., A-12, 2nd Floor, Sector 2, Noida-201301 UP, India 
the efficacy of triclofos). Nonetheless, there were no studies that directly compared the efficacy of melatonin with that of triclofos. Hence, the authors attempted to perform an indirect comparison of melatonin and triclofos by collecting pooled estimates of all studies that individually described their safety and efficacy. The demographic characteristics and the clinical parameters were comparable between both groups.

Ramsay's sedation score of 4 was considered as an adequately sedated state for performing EEG in most of the studies that were included in the meta-analysis. Triclofos group showed around 90\%, whereas the melatonin group showed $76 \%$ of sedation and successful recording of EEG, with a $p$-value of 0.058 . However, in $12 \%$ and $22 \%$ of triclofos and melatonin groups, respectively, adequate sedation could not be achieved, and the procedure had to be differed. The triclofos group showed statistically insignificant higher adverse effects such as increased duration of sleep, excessive drowsiness, bradycardia, and hypotension, whereas children who were prescribed melatonin showed minimal to no ADEs. The successful sedation rate estimated by sleep onset latency and percentage of epileptiform abnormalities detected in EEG was also comparable between both groups. This implication of the research is valuable for the clinical application while planning the sedative of choice for children with developmental abnormalities.

The results of this systematic review suggested that both the drug groups were efficacious in achieving adequate sedation for facilitating EEG in uncooperative children. However, melatonin showed better tolerance and relatively fewer side effects when compared with triclofos. The only disadvantage of the administration of melatonin was the need for a second dosage to maintain the sleep state. Triclofos, a chloral hydrate derivative, had a longer duration of action. Therefore, a clinician has to keep in mind the pros and cons of both the drugs and the duration taken for the procedure to be completed before choosing either of the drugs.

Although no randomized controlled trial (RCT) was available at the time of conducting this systematic review and meta-analysis, Lalwani et al had compared the efficacy and adverse effects profile of melatonin and triclofos in a recently published RCT. ${ }^{10}$ They included 228 children (114 each received melatonin and triclofos) to compare their efficacy and tolerability. The proportion of successful EEG was $89.4 \%$ and $91.2 \%$ in melatonin and triclofos group, respectively, and the difference was nonsignificant. Similarly, other results including the efficacy of the first dose (64\% vs. $63.15 \%$ ), the need for the augmentation dose ( $25.4 \%$ vs. $28 \%$ ), mean total sleep duration ( 80 minutes vs. 82.39 minutes), and adverse effects $(6.14 \%$ vs. $8.65 \%)$ were also comparable in both groups. This RCT also supports the findings of this metaanalysis that the efficacy and tolerability of melatonin and triclofos are almost the same and both can be safely used to achieve sleep for EEG in children. ${ }^{10}$

There is enough evidence that validates the use of melatonin and triclofos as sedatives for sleep-induced EEG; however, there is a lack of research that claims the superiority of one drug over the other. This meta-analysis could not conclude if melatonin or triclofos is an ideal choice of sedative for children. This ambiguous interpretation of the outcome steers to further confusion during practical application. The advantages these drugs have over each other, as hinted by this systematic review and meta-analysis, cannot be substantiated based on the indirect comparison of the pooled estimates alone. Hence, in my opinion, this could be considered as a shortcoming of this systematic review and meta-analysis. To overcome this, more long-term RCTs should be conducted comparing the safety, efficacy, onset, and duration of action of both drugs. Attempts to assimilate the outcomes of multiple RCTs will minimize the amount of conflict in the interventions being reviewed.

\section{Authors' Contributions}

A.A. contributed in drafting the article, reviewing literature, editing of the manuscript, and revising the article critically for important intellectual content. A.A. acted as guarantor.

\section{Funding \\ None.}

Conflict of Interest

None declared.

\section{References}

1 Coté CJ, Wilson SAmerican Academy Of Pediatrics American Academy of Pediatric Dentistry. Guidelines for monitoring and management of pediatric patients before, during, and after sedation for diagnostic and therapeutic procedures. Pediatrics 2019; 143(06):e20191000

2 Scottish Intercollegiate Guidelines Network. SIGN Guideline 58: safe sedation of children undergoing diagnostic and therapeutic procedures. Paediatr Anaesth 2008;18(01):11-12

3 Karasek M, Winczyk K. Melatonin in humans. J Physiol Pharmacol 2006;57(05, Suppl 5):19-39

4 Arendt J. Melatonin: characteristics, concerns, and prospects. J Biol Rhythms 2005;20(04):291-303

5 Blackmer AB, Feinstein JA. Management of sleep disorders in children with neurodevelopmental disorders: a review. Pharmacotherapy 2016;36(01):84-98

6 Grigg-Damberger M, Ralls F. Treatment strategies for complex behavioral insomnia in children with neurodevelopmental disorders. Curr Opin Pulm Med 2013;19(06):616-625

7 Wassmer E, Carter PF, Quinn E, et al. Melatonin is useful for recording sleep EEGs: a prospective audit of outcome. Dev Med Child Neurol 2001;43(11):735-738

8 Jackson EA, Rabbette PS, Dezateux C, Hatch DJ, Stocks J. The effect of triclofos sodium sedation on respiratory rate, oxygen saturation, and heart rate in infants and young children. Pediatr Pulmonol 1991;10(01):40-45

9 Panda PK, Panda P, Dawman L, Sharawat IK. Systematic review and meta-analysis of efficacy and safety of melatonin and triclofos for inducing adequate sedation for sleep EEG in children. J Neurosci Rural Pract 2021 (e-pub ahead of print). Doi: 10.1055/s-00411736511

10 Lalwani S, Srivastava K, Thakor B, et al. Efficacy and tolerability of Melatonin vs Triclofos to achieve sleep for pediatric electroencephalography: a single blinded randomized controlled trial. Eur J Paediatr Neurol 2021;34:14-20 